Ambient Science, 2016: Vol. 03(Sp1); 84-85

DOI:10.21276/ambi.2016.03.sp1.nn03

ambient

SCIENCE

Vol. 3(Sp1):84-85

Year 2016

NOTES ON NATURAL HISTORY

\title{
Occupational Causes Related Male Infertility: A Case Study in Iran
}

\section{Rasekhjahromi Athar', Asadi Mahdi' ${ }^{2 *}$, Ghanei Masoud ${ }^{3}$, Maalhagh Mehrnoosh ${ }^{4}$, Alipour Mohammad Hossein ${ }^{5}$, Sanie Mohammadsadegh', Alipour Farzaneh', Hosseinpoor Masoumeh ${ }^{1}$}

'Obstetrics and Gynecology Department, Jahrom University of Medical Sciences, Jahrom, Iran

${ }^{2}$ Department of Emergency Medicine, Jahrom University of Medical Sciences, Jahrom, Iran

${ }^{3}$ Anesthesia and Intensive Unit Care Department, Jahrom University of Medical Sciences, Jahrom, Fars, Iran

${ }^{4}$ Student Research Committee, Shiraz University of Medical Sciences, Shiraz, Iran

${ }^{5}$ Student Research Committee, Razi-University, Kermanshah, Iran

\section{Introduction:}

Infertility is def ined as an inability of couples to conceive a clinical pregnancy even after the 12 months or more of unprotected intercourse or therapeutic donor insemination (PCASRM, 2013). The man should be evaluated concurrently with the woman, since a male factor is the primary or contributing cause in $40 \%$ to $60 \%$ of cases (Schlegel, 2009). As the WHO, the prevalence of infertility is $10-15 \%$ which means that one out of six couples is suffering from infertility, among which $35-40 \%$ of cases are related to male infertility disorders and $20 \%$ related to couple factors. Thus, it could be concluded that male causes play an important role in infertility cases (Vaziri et al., 2011).

Generally, in the initial screening of male partner of the infertile couple, a reproductive history, physical parameters and analysis of at least one semen sample is taken which includes; semen volume (normal, 1.5-5 mL), semen quality, sperm density (normal, >20 million sperm $/ \mathrm{mL}$ ), sperm motility (normal, $>60 \%$ of sperm having normal movement), sperm morphology ( $>60 \%$ of sperm should be normal, and less than 2-3\% should be immature) (Bouloux et al., 2002). If the initial evaluation was found abnormal, the complete evaluation the case is referred to the urologist or male reproductive specialist (Sheiner et al., 2003). The patient's medical history could indicate the risk factors and lifestyles which could have effects on male infertility. Many factors could affect semen quality, so assessments must be done in order to exclude the abnormal anatomy, genetic causes, and endocrinology disturbance, history of testicular trauma, surgery, or mumps. Heat exposure, sedentary life, extended travels, extreme allergic reactions, drug dependence, alcohol abuse, heavy smoking, medications such as cimetidine, spironolactone, nitrofuran, sulfasalazine, erythromycin, exposure to
Study Area:Jahrom, Iran

Coordinates: $28^{\circ} 30^{\prime} \mathrm{oo}^{\prime \prime} \mathrm{N} ; 53^{\circ} 33^{\prime} 38^{\prime \prime} \mathrm{E}$

Key words: Physical exercise, Psychological factors, Back pain

Approval: The study was initiated after approval of research and ethics committee of Jahrom University of Medical Sciences, after clarifying the study protocol, consent was written by participants.

environmental and industrial toxin are some of the risk factors causes male infertility.(Carlsen et al., 1979; Jurewicz et al., 2009; Ossar, 1984). In recent decades, the increases in environmental pollution in industrial based countries have also increased infertility. Approximately 104,000 chemical and physi-agents existing in workplaces were already listed at the National Institute for Occupational Safety and Health Registry. The toxicity of most of these materials has been studied in humans; exposure to some of these agents may cause reproductive abnormalities (Gold et al., 1994).

\section{OurStudy:}

We conducted a descriptive analytical study on 106 men who referred to the infertility clinic in Jahrom city in South of Iran in between March 2013 to February 2015.

Participants were referred to the pathobiology laboratory for minimum two semen analysis after tabulating their proper medical history and physical examination. Semen samples were collected according to the WHO guidelines. The samples were collected by condom or substandard plates and participants who had anatomical or medical diseases were excluded from the study. Semen analysis was done on the basis of WHO's guideline to obtain volume, $\mathrm{PH}$, vitality, sperm concentration, motility and morphology (Cooper, 2010).

\section{Our Findings:}

We divided the 106 married infertile men (participants) into two groups on the basis of their work exposure or not exposure to various worthy materials (Figure-1). The average age was noted as $30.3 \pm 4.8$. How far marital life was concerned, $9.1 \%$ of the total were enjoying married life since one year, $40.9 \%$ for three years, and $50 \%$ more than three years. We found total $42(39 \%)$ participants were having the normal semen analysis, from remaining 64 
participants who had abnormal semen analysis 25 (39\%) had no exposure. Further, we correlated the occupation of the participants with their respective sperm count and motility which has been given in Table-1.

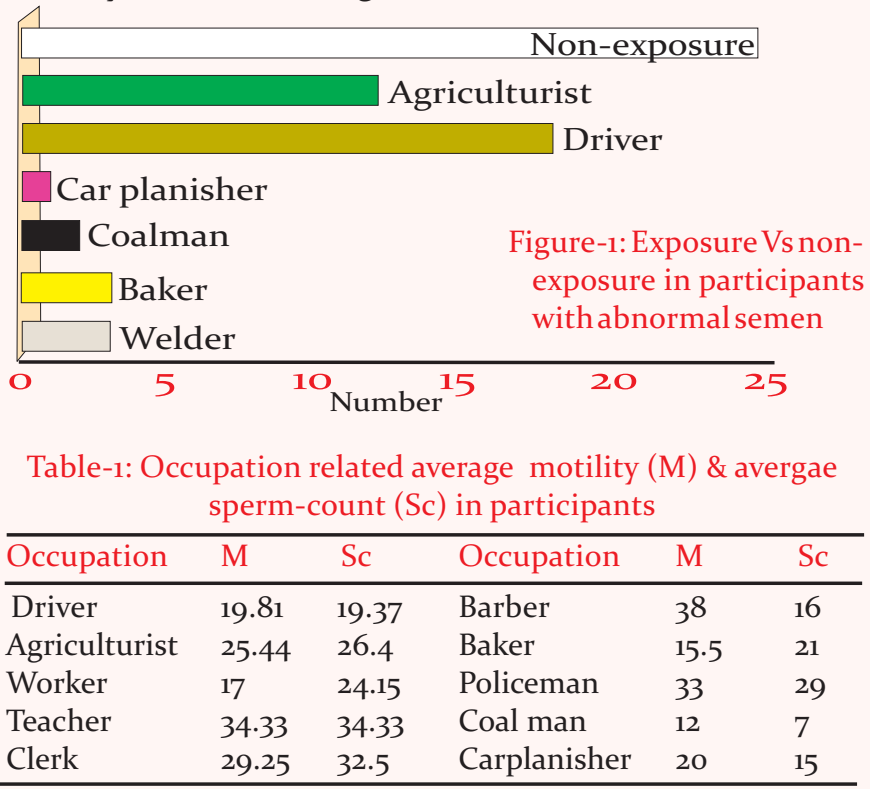

\section{Discussion:}

We found that certain working hazards affect the human reproductive system. It is well established that heat reduces sperm production. The median value of scrotal skin temperature was $33.3^{\circ} \mathrm{C}$ in the daytime and $34.8^{\circ} \mathrm{C}$ at night (Hjollund et al., 200o). It has already established that the heat is an independent factor for male infertility or 4.5, 95\%CI 1.9-10.6 (Sheiner et al., 2003). These results resemble our findings regarding the association of heat and male infertility. Further, Vaziri et al. (2011) revealed a close association between occupation and sperm motility. The agricultural occupations or exposure to pesticides have also reported to impaired the fertility (Vaziri et al., 2011). As per the recent research, chemicals may act as antiandrogens, inhibitors of steroidogenic pathways as well as oestrogens or antiestrogens (Jeng, 2014). Dibromochloropropane (DBCP) is an agricultural pesticide that affects human spermatogenesis. It has been well established that the agent could cause permanent azoospermia. Exposure to a variety of agricultural pesticides has also been linked to impaired semen quality. Recent research has demonstrated that chemicals may act as antiandrogens, inhibitors of steroidogenic pathways as well as oestrogens or antiestrogens (Sifakis et al., 2011) these results also strengthens our findings. The literature review indicates that occupations which involve frequent contacts with heavy metals (lead magnesium), pesticides, solvents and other substances which have the estrogenic effect, can contribute to the decrease in male fertility (Sheiner et al., 2003). Such agents may reduce sperm production. The spermatogenic cycle spans are 72 days in humans and can be disrupted by toxicants at any stage of cell differentiation, giving rise to reduced sperm counts, morphologically abnormal sperm, and impaired stability of sperm chromatin or sperm DNA damage (Jensen et al., 2006). Toxins accumulating in the epididymis, prostate, vesicular seminal or seminal fluid may impair normal progressive sperm motility (Sengupta, 2014).These studies are consistent with our findings regarding effect of pesticides in agriculture on male infertility. We found the most disorders of semen analysis were in motility then, functional sperm disorders. Taken together we clarif ied in our study that some occupations like agricultures, drivers and workers might be more vulnerable for male infertility.

Acknowledgements:

Authors are thankful to all the participants who cooperated in this study.

\section{References:}

Bouloux, P., Warne, D.W. \& Loumaye, E. (2002): Efficacy and safety of recombinant human follicle-stimulating hormone in men with isolated hypogonadotropic hypogonadism. Fertil Steril., 77(2):270-273.

Cooper, T.G., Noonan, E., von Eckardstein, S., Auger, J., Baker, H.W., Behre, H.M., Haugen, T.B., Kruger, T., Wang, C., Mbizvo, M.T. \& Vogelsong, K.M. (2010): World Health Organization reference values for human semen characteristics. Hum. Reprod. Update, 16:231-245..

Gold, E.B., Lasley, B.L. \& Schenker, M.B. (1994): Reproductive hazards-State of the art reviews-Introduction: Rational for an update. Occup. Med., 9:363-72.

Hjollund, N.H., Bonde, J.P., Jensen, T.K. \& Olsen, J. (2000): Diurnal scrotal skin temperature and semen quality. The Danish First Pregnancy Planner Study Team. Int JAndrol., 23(5):309-318.

Jeng, H.A.(2014), Exposure to Endocrine Disrupting Chemicals and Male Reproductive Health. Front Pub. Health.; 2: 55 .

Jensen, T.K., Bonde, J.P. \& Joffe, M. (2006): The influence of occupational exposure on male reproductive function. Occup.Med., 56 (8):544-553.

Jurewicz J, Hanke W, Radwan M, Bonde JP, (2009). Environmental factors and semen quality. Int. J. Occup. Med. Environ. Health, 22(4):305-329.

Osser S, Ledholm OP \& Ranstam J (1984) Depressed semen quality: a study over two decades. Arch Androl., 12:113-116.

PCASRM (2013): Definitions of infertility and recurrent pregnancy loss: a committee opinion. Ferti. Steril., 99(1), 63.

Schlegel, P.N. (2009): Evaluation of male infertility. Minerva Ginecol., 61(4):261-83.

Sengupta, P. (2014), Metals and male reproduction: The possible mechanisms. Adv. Biomed. Res., 3:129

Sheiner, E.K., Sheiner, E., Hammel, R.D., Potashnik, G., \& Carel, R. (2003): Effect of occupational exposures on male fertility: literature review. Indust. health, 41(2):55-62.

Sifakis, S., Mparmpas, M., Soldin, O.P. \& Tsatsakis, A.(2011): Pesticide Exposure and Health Related Issues in Male and Female Reproductive System. In Pesticides - Formulations, Effects, Fate, ed. by M. Stoytcheva. Pub.: Intech, Opeaccess.

Vaziri, M.P.H., Gilani, M.A.S. \& Kavousi, A. (2011): The Relationship between Occupation and Semen Quality. Int J Fertil Steril., 5(2): 66-71. 